\title{
Metadatos y descripción como representación del contexto archivístico
}

Metadata and description as a representation of archival context

\author{
Alejandro Delgado Gómez \\ Archivo Municipal de Cartagena. Plaza del General López Pinto s/n. \\ 30201-Cartagena. alejandro@ayto-cartagena.es
}

\begin{abstract}
Resumen
La ponencia informa acerca de un trabajo de investigación en curso, cuyo objetivo es mostrar cómo el documento de archivo obtiene calidad de evidencia mediante la representación de un adecuado contexto de creación, gestión y uso obteniéndose esta representación mediante el uso de esquemas complejos de metadatos. Para argumentar esta hipótesis, se explora el discurso archivístico contemporáneo, y se explica la diferencia entre el documento como objeto y el documento como proceso. Se analizan varios modelos de metadatos. Además, se describe un escenario donde verificar la posibilidad de aplicación de un esquema de metadatos multidimensional. Se concluye con la propuesta de una segunda fase, que aborde el proceso de la descripción archivística a partir de un modelo concéntrico de sistemas de conocimiento, información y documentos.
\end{abstract}

Palabras clave: Archivos. Contexto archivístico. Documento archivístico. Metadatos. Modelo enfocado al documento. Modelo enfocado al proceso. Esquema de metadatos.

\section{Introducción}

Por utilizar la acertada expresión de Sue McKemmish (1996), el hombre es un animal cuentacuentos. Aquellos cuentos que el hombre considera relevantes se fijan en documentos, con independencia de la noción de documento que cada individuo o grupo adopte. En el marco de la tradición occidental, se entiende por documento información registrada sobre un soporte estable, pero el tipo de información, los soportes, la percepción de la estabilidad y el modo de registro están determinados culturalmente. Sea cual sea la noción de documento que se adopte, para todo individuo o grupo social más o menos organizado, existe un subconjunto de documentos, de los que se considera que constituyen evidencia de acciones, y que se utilizan a efectos de responsabilidad y memoria, viniendo determinadas también las nociones de evidencia, responsabilidad y memoria culturalmen-

\begin{abstract}
This paper reports about a research project, with the aim of showing how a record obtains its quality of evidence by representing a sufficient context of creation, management and use. This representation is obtained by using complex metadata schema. In order to argue this hypothesis, we explore the modern archival discourse, and explain the difference between the record as an object and the record as a process. We analyse several metadata models. In addition, we describe a setting where we will be able to test the possibility of applying a multidimensional metadata schema. We recommend a proposal for a second step, which will address the process of the archival description from a concentric model of knowledge, information and documents/records systems.
\end{abstract}

Keywords: Archives. Archival context. Archival document. Document-focused model. Metadata. Metadata schema. Process-focused model. Record.

te. Este subconjunto de documentos se conoce como documentos de archivo.

Se conceptualicen como se conceptualicen estas nociones, existe un elemento que diferencia los documentos de archivo de otros tipos de documentos. En el caso de los documentos de archivo, la evidencia de acciones viene dada, no sólo por el contenido informativo del documento, sino de manera muy especial por la adecuada representación del contexto en que se creó, gestionó y utilizó. Esto sería cierto, no sólo en el actual entorno digital, en el que por su mismo carácter difuso y complejo es esencial para identificar adecuadamente objetos similares a documentos que no están auto-contenidos, sino también en cualquier entorno de generación de documentos. En el discurso archivístico que se construye entre mediados del siglo diecinueve y mediados del veinte, la representación de este contexto era relativamente sencilla, debido a 
factores muy diferentes que podrían incluir el uso de unas tecnologías discretas para la creación, gestión y utilización de documentos; la existencia de burocracias altamente jerarquizadas, verticales y lineales; la utilización de constructos sociales bien delimitados mediante rigurosos mecanismos de exclusión; o el apoyo en una teoría científica positivista que permitía la fácil categorización del universo. Se podría argumentar que este conjunto de factores, que permitieron una eficaz gestión de documentos durante algo más de un siglo, comienza a desaparecer en las dos últimas décadas del siglo veinte, y puede darse por finiquitado a comienzos del siglo veintiuno: las tecnologías de la información y de las comunicaciones ya no son discretas; las burocracias son cada vez más horizontales y difusas; el discurso social ha incorporado las voces de los excluídos; y los constructos científicos positivistas han sido fuertemente cuestionados hasta concluir en el actual constructo científico postmoderno.

Finiquitado el modelo en el que se apoyaba, la archivística de las tres últimas décadas se ha visto obligada a repensar todas sus asunciones conceptuales y todos sus métodos de trabajo, incluído el modo en que representaba el contexto mediante la descripción archivística, y la validez de los principios en que se apoyaba esta práctica de la descripción, a saber, el principio de procedencia y el principio de orden original. La archivística postmoderna no cuestiona, por supuesto, la necesidad de la descripción; pero, de acuerdo con una noción de documento que se orienta más al proceso que al objeto, la enriquece, al incorporarla a un proceso continuo de asignación de metadatos, de los que la descripción, tal y como se concebía en la archivística occidental, sería un subconjunto. De igual modo, no discute la eficacia del principio de procedencia, sino la eficacia de la manera en que se ha conceptualizado la procedencia, identificándola de manera unívoca con un objeto "fondo" que también ha sido sometido a discusión. Finalmente, no discute el principio de orden original, en el que de hecho se apoya fuertemente para elaborar otros modelos descriptivos; simplemente admite la posibilidad de que, con independencia de que el orden original permita gestionar eficazmente documentos en el entorno de trabajo del archivero, existen otros entornos que escapan al control del archivero y en los que el documento puede ser reodernado de múltiples maneras y dependiendo de diferentes necesidades.

Un contexto complejo de creación, gestión y uso de los documentos requiere un modelo complejo de representación del mismo. Tradicionalmente, la descripción archivística, a partir del argumento de que los documentos de archivo son únicos y por tanto cada archivo constituye un mundo, no estaba sujeta a normalización. A pesar de que otros discursos archivísticos ya estaban trabajando en el asunto de la normalización de la descripción, el discurso archivístico mayoritario en Occidente siguió utilizando el criterio de la imposibilidad de la normalización, hasta la aparición de la norma, publicada por el Consejo Internacional de Archivos (ICA), Norma Internacional de Descripción Archivística (ISAD(G)). Desde la primera edición de la norma $\operatorname{ISAD}(\mathrm{G})$ se ha producido un vuelco en el pensamiento archivístico respecto a la descripción, tanto por la propia reflexión acerca de la norma, como por la irrupción de esfuerzos paralelos y en algunos casos implícita o explícitamente disconformes, así como por la recuperación y expansión de aquellos otros discursos que ya reflexionaban acerca del problema de la descripción. En la actualidad, por tanto, existe un amplio cuerpo normativo relativo a la descripción archivística. Parte de este cuerpo normativo se inscribe en el marco de la archivística custodial tradicional, que sigue considerando sin modificaciones la procedencia como el acto de identificar un agente con un fondo, y el documento como un objeto sobre el que se actúa en diferentes momentos independientes entre sí. Sin embargo, también están emergiendo ricos modelos de metadatos que presumen que la representación del contexto no es tanto asunto de descripción de las convencionales entidades de procedencia, cuanto, especialmente, un proceso permanente de asignación de relaciones, y de descripción de estas relaciones; de igual modo, asumen que el documento ya no es más, o ya no es sólo, un objeto, sino, sobre todo, el resultado de un proceso, tanto como el proceso mismo. Parafraseando a Terry Cook (1994), asumen que por detrás del objeto documento está el proceso de documentar.

En lo que sigue, se describen algunos de los modelos de metadatos de archivo actualmente en uso. No es nuestra pretensión ser exhaustivos, pero sí recoger al menos aquellos que marcan las pautas y tendencias más generalizadas. Así, en primer lugar, se expone el modelo de metadatos, abarcador pero no neutral con respecto al paradigma, de la norma ISO 23081 -Metadatos para la gestión de documentos. A continuación, se examinan algunos de los schemas de metadatos asociados a la noción de documento como objeto -a saber, Norma Internacional de Descripción Archivística $(\operatorname{ISAD}(\mathrm{G}))$, y Encoded Archival Description (EAD). Posteriormente, se examinan del mismo modo algunos de los schemas de metadatos 
asociados a la noción de documento como proceso -a saber, el llamado sistema australiano de serie, el Australian Recordkeeping Metadata Schema (RKMS) y el schema derivado del proyecto Clever Recordkeeping Metadata. De este análisis se espera poder concluir que este segundo tipo de modelo permite una rica representación del contexto en entornos complejos, superando así las deficiencias de los modelos custodiales.

\section{Descripción de schemas de metadatos de archivo actualmente en uso}

\subsection{ISO $23081-$ Metadatos para la gestión de documentos}

ISO 23081 es consecuencia de la publicación y la progresiva implantación de la norma ISO 15489 Information and documentation-Records management. La norma de gestión de documentos insiste en varios lugares en la importancia de los metadatos para la creación, gestión y uso de buenos documentos, de tal manera que la redacción de una norma acerca de tales metadatos de gestión de documentos parece un resultado natural. La norma 23081 se divide en dos partes ya publicadas y otras dos propuestas. De acuerdo con la propia norma

ISO 23081 es una guía para comprender, implantar y utilizar metadatos dentro del marco de ISO 15489, Information and documentation - Records management. Aborda la relevancia de los metadatos de gestión de documentos en los procesos, y los diferentes roles y tipos de metadatos que apoyan actividades y procesos de gestión de documentos. También establece un marco para gestionar esos metadatos. No define la implantación de una serie obligatoria de metadatos de gestión de documentos, dado que estos metadatos diferirán en sus detalles de acuerdo con requisitos organizativos o específicos de la jurisdicción. Sin embargo, pondera las principales series de metadatos existentes, de acuerdo con los requisitos de ISO 15489. La norma establece un marco para crear, gestionar y utilizar metadatos de gestión de documentos y explica los principios que la regulan (ISO 23081, 2006).

La norma ha hecho un notable esfuerzo por reconciliar diferentes tradiciones, admitiendo el uso de modelos de metadatos tanto planos como multidimensionales. En línea con ISO 15489 , la norma discrimina metadatos y descripción archivística. Sin embargo, en la tradición archivística española, la discriminación archivo/gestión de documentos no existe, y, por tanto, tampoco la paralela discriminación descripción archivística/metadatos. En la presente exposición se sigue, pues, el ya mencionado criterio de considerar la descripción como un subconjunto del proceso continuo de asignación de metadatos.

ISO 23081 no sólo identifica metadatos para los documentos, tal y como haría un modelo custodial, sino también, como subconjuntos de los metadatos para documentos, metadatos para la accesibilidad y la seguridad de éstos; y metadatos para mandatos, agentes, procesos y procesos de gestión de documentos, en línea con la norma fuente Recordkeeping Metadata Schema (RKMS), que se describe más adelante.

De igual modo, ISO 23081 identifica, significativamente, metadatos en el punto de captura, es decir, en el momento de incorporar un documento a un sistema, y metadatos posteriores al punto de captura, que se asignan en un proceso continuo de gestión y uso, sin dejar de reutilizar los metadatos del punto de captura.

\subsection{Norma Internacional de Descripción Archivística (ISAD(G))}

$\operatorname{ISAD}(\mathrm{G})$ fue el primer esfuerzo por llegar a unos principios normalizados que sirvieran de base para descripciones homogéneas a nivel internacional. La primera edición, fue aprobada en 1993, y, en lo que concierne a la presente exposición, adoptaba sin titubeos la noción de fondo como fundamental para la descripción archivística, así como la identificación de procedencia y creador único. Así, en el mismo glosario de términos, bajo la entrada "Creador" se indica "véase Procedencia", y ésta se define como "la organización o individuo que creó, acumuló y/o mantuvo y utilizó documentos en la ejecución de actividades personales o corporativas". Puesto que "Fondo" se define como "el total de documentos, con independencia de la forma o soporte, orgánicamente creados $y / o$ acumulados y utilizados por una persona particular, familia, o cuerpo corporativo en el curso de las actividades y funciones de ese creador" (International Council on Archives, 1993), el proceso de describir se convierte en una identificación circular creador-fondo y fondo-creador, que el autor de la presente exposición ya ha criticado en otros lugares (Delgado Gómez 2006).

La segunda edición de la norma, aprobada en 1999, suaviza los términos, si bien más en la forma que en el fondo. Así, por ejemplo, la entrada "Creador" ya no se identifica con la entrada "Procedencia", aunque se define "creador" del mismo modo que en la primera edición se definía "procedencia", y la definición de "fondo" como nivel de agregación fundamental persiste. "Procedencia", en la segunda edición, se define como la relación unívoca entre un creador y un fondo: "la relación entre los documentos y las 
organizaciones o individuos que los crearon, acumularon y/o mantuvieron y utilizaron en la ejecución de actividades personales o corporativas" (International Council on Archives, 2000). El contexto, tal y como se concibe en $\operatorname{ISAD}(G)$, viene dado por descripciones individuales, realizadas mediante la norma hermana International Standard Archival Authority Record for Corporate Bodies, Persons and Families (ISAAR(CPF)), de entidades creadoras, con las que los documentos o sus agregaciones se relacionan uno-a-uno. En ningún caso es posible vincular, tal y como sugiriera Chris Hurley (1995), "múltiples entidades contextuales con múltiples entidades de gestión de documentos de múltiples maneras".

Por lo demás, en fecha tan reciente como 2006, el ICA ha publicado un borrador de norma para la descripción de funciones (International Council on Archives, 2006). El borrador reconoce la importancia de las funciones y actividades para la descripción, y asume su lectura de ISO 15489 y otros documentos relacionados con el análisis funcional. De manera muy significativa, en la definición de procedencia se ha incorporado una nueva línea, que extiende la definición anterior: "Procedencia es también la relación entre los documentos y las funciones/actividades que los produjeron" (International Council on Archives, 2006). No obstante, y a pesar de los síntomas de que el ICA, y debe entenderse que también la comunidad internacional, está modificando su perspectiva, en el borrador las funciones siguen siendo contingentes con respecto a las entidades creadoras, cuando lo cierto es que las entidades creadoras son contingentes con respecto a las funciones.

\subsection{Encoded Archival Description (EAD)}

EAD es "una norma de codificación en Standard Generalized Markup Language (SGML) diseñada específicamente para marcar información contenida en instrumentos de descripción archivísticos" (Corneau, 1998). EAD no es, en sentido estricto, un lenguaje de descripción de materiales archivísticos, sino un lenguaje de codificación, y, en esta medida, destinado a ser interpretado por máquinas para devolver una visualización legible por humanos; es decir, es un lenguaje para codificar instrumentos de descripción, no los materiales archivísticos en sí mismos.

Tal y como indicaba Daniel V. Pitti (1997), EAD nació como un medio para superar las deficiencias del formato MARC para las descripciones archivísticas. Dos años más tarde, el mismo autor explicaba la oportunidad de la normalización en términos de explotación de tecnologías abiertas a efectos de regularización del caos y de explotación de los recursos sobre la web, representando la descripción archivística en un entorno informático (Pitti 1999).

Es evidente que la intención de los creadores de EAD es diseminar instrumentos de descripción en Internet, aunque el lenguaje se ha utilizado también con otros propósitos. En este sentido, va un paso más allá de $\operatorname{ISAD}(G)$. Sin embargo, debe tenerse en cuenta que la tradición norteamericana, en donde nace EAD, discrimina de manera muy vigorosa la gestión de documentos de la archivística, un territorio menor habitado por colecciones o fondos de valor histórico. A este respecto, resulta significativo que EAD fuera una respuesta a las insuficiencias de MARC, utilizado sólo para las colecciones históricas, en conjunción con la norma de contenido APPM (Archives, Personal Papers and Manuscripts). De igual modo, EAD se ha asociado a la más reciente norma de contenido DACS (Describing Archives: A Content Standard), que adopta una perspectiva fuertemente custodial, tal y como se expresa en los llamados Principios Custard.

No obstante, pese a su orientación fuertemente custodial, EAD, junto con el modelo paralelo Encoded Archival Context (EAC), para el etiquetado normalizado de personas, familias y entidades, cumple la función cultural de diseminar colecciones históricas en Internet, y se ha utilizado, de manera enriquecedora, para fines que se alejan del concepto de fondo.

\subsection{Sistema de serie}

El sistema de serie es un método para describir documentos, así como sus contextos de creación y gestión, a lo largo del tiempo. El sistema fue desarrollado en los años sesenta del siglo veinte, en el ámbito de la archivística australiana, y se distingue de otras prácticas descriptivas por el hecho de que no toma como unidad fundamental el fondo asociado a una sola procedencia, sino, como su nombre indica, la serie, vinculada a múltiple información contextual. Sin embargo, puede aplicarse a niveles de agregación diferentes a la serie. Además, el sistema de serie separa la descripción del documento de la descripción de su contexto, de tal modo que los documentos, los agentes creadores y las funciones son entidades descriptivas distintas. Las descripciones individuales de cada una de estas entidades se vinculan para devolver una representación más completa, rica e informativa, de los documentos, su contexto y su gestión a lo largo del tiempo. 
Se considera que el padre del sistema de serie es Peter Scott, que desarrolló el sistema a partir de 1964, sugiriendo dos marcos de descripción: el contexto o la procedencia, y la gestión de documentos, que podían ponerse en relación de tal manera que quedara indicado con claridad cuántos, y durante cuánto tiempo, eran los creadores de un determinado tipo de documento. Desde 1964, el sistema de serie ha evolucionado en diversos sentidos, de los que no sería el menor, por ejemplo, el hecho de que en la actualidad se consideren también como entidades contextuales, no sólo las organizaciones y las personas, sino también las funciones y actividades. Por su parte, el especialista Chris Hurley ha elaborado, a partir del sistema, un complejo y rico procedimiento descriptivo que incluye los conceptos de función ambiente, de procedencia múltiple y, en los últimos años, también el concepto de procedencia paralela (Hurley 2004).

En la presente exposición se describe el procedimiento elaborado por el Committee on Descriptive Standards (CDS) de la Australian Society of Archivists (ASA), que reconoce diferentes tipos de entidad, bien entendido que éstas no son obligatorias: entidad ambiente (organizaciones, familias, funciones), o entidad que proporciona contexto a las entidades de procedencia; entidad de procedencia (agencias, personas, familias, actividades), o entidad que crea, genera, mantiene, utiliza, controla o dispone de los documentos; entidad de gestión de documentos (series), o agregación de documentos, que no tiene por qué ser una serie; puede ser todo un archivo, un expediente o si es útil un fondo; y entidad de contenido (items), o unidad individual, como un documento dentro de un expediente, una fotografía, un plano; pero también, dependiendo del sistema de implantación, un expediente, una caja, etc.

El sistema de serie se apoya en la descripción de relaciones contextuales entre las entidades mencionadas, así como en la definición y descripción de las relaciones mismas. La descripción de entidades y relaciones entre ellas se realiza mediante la asignación de elementos de metadatos, que especifican la información que debe ponerse en combinación para conformar la descripción de una entidad; proporcionan estructura y significado a la descripción de la entidad; y facilitan la indización y la búsqueda de elementos. A cada entidad identificada en el sistema de serie se le asignan unos determinados elementos de metadatos normalizados, pero éstos no son obligatorios, y pueden elegirse aquellos que tengan sentido de acuerdo con las necesidades descriptivas de un archivo.

\subsection{Australian \\ Recordkeeping \\ Metadata Schema (RKMS)}

RKMS constituye una evolución con respecto al sistema de serie. Desarrollado por el Records Continuum Research Group en el marco del proyecto SPIRT (Recordkeeping Metadata Research), es finalidad de RKMS

desarrollar un conjunto normalizado de elementos estructurados de metadatos para la gestión de documentos; un marco para desarrollar y especificar normas de metadatos de gestión de documentos; y un marco para leer o mapear conjuntos de metadatos de tal modo que se haga posible su interoperabilidad semántica, estableciendo equivalencias y correspondencias que puedan proporcionar la base para la traducción semiautomatizada entre schemas de metadatos (McKemmish et al., 1999).

RKMS concibe los documentos como "participantes activos en los procesos y tecnologías, más que objetos pasivos a ser descritos retrospectivamente" (Reed, 1997). Los documentos, por tanto, son "objetos dinámicos que tienen que asociarse a lo largo de su rango de vida con capas cada vez más amplias y ricas de metadatos contextuales para mantener su fiabilidad y autenticidad, y para que sean significativos y accesibles a lo largo del tiempo y el espacio" (McKemmish et al., 1999).

Los metadatos de gestión de documentos se definen de modo que puedan incluir el máximo de información normalizada que identifique, autentique, describa, gestione y haga accesibles los documentos creados en el contexto de actividades sociales y organizativas. Los metadatos RKMS identifican y describen tanto los documentos que reflejan esas actividades, como las características significativas de los contextos en que los documentos se crean, gestionan y utilizan, a saber: las personas o agentes que participan en la creación, control, gestión y uso de los documentos; las funciones, actividades y transacciones que desarrollan estas personas $o$ agentes; y las funciones, actividades y transacciones de gestión de documentos.

El conjunto de metadatos RKMS se apoya en un complejo marco conceptual que reconoce diferentes tipos de entidad: entidades de actividad, entidades de agente o persona, entidades de documento, y entidades de gestión de documentos. De igual modo, reconoce las relaciones entre entidades y los mandatos que regulan las entidades y sus relaciones. Para cada una de estas entidades, así como para sus relaciones y mandatos, se facilitan series específicas de metadatos, de uso variable. Debe tenerse en cuenta, en cualquier caso, que las relaciones, 
para RKMS, no tienen por qué ser binarias, y de hecho raramente lo son.

\subsection{Clever Recordkeeping Metadata}

El proyecto Clever Recordkeeping Metadata va un paso más allá con respecto a RKMS, al incorporse de lleno a los entornos web y reimaginar sistemas de archivo colectivos, multidimensionales, paralelos, relacionados de manera múltiple, y relacionados con los procesos mismos de gestión de documentos (Evans et al., 2005).

El proyecto aborda el problema de la generación automática de metadatos en diferentes sistemas de creación, gestión y uso de documentos, metadatos cuya producción actual consume recursos y es específica de la aplicación. Sin embargo, gran parte de la información contextual, o metadatos, necesaria para la gestión de documentos ya se genera en otras aplicaciones de las organizaciones; pero estos metadatos no se están reutilizando en la gestión de documentos de la administración, el gobierno y la empresa, a pesar de que existen herramientas automatizadas de modelado, marcado y sintaxis que lo permiten.

El proyecto postula que es posible reutilizar los metadatos generados en los sistemas de creación en beneficio de los procesos archivísticos, lo que expresa vigorosamente mediante la fórmula, por la que ha llegado a ser conocido, "crear una vez, utilizar muchas veces". Con este fin, el proyecto desarrolla un prototipo centrado en el usuario, que demuestra el modo en que este tipo de meta-herramientas debe contribuir a la eficacia y la eficiencia en la gestión de metadatos de documentos de archivo. El modo en que tales eficacia y eficiencia se manifiestan viene dado por el uso de los estándares XML y SOAP, que deben facilitar la interoperabilidad de diferentes series de metadatos, distribuídos en diferentes capas de abstracción.

\section{Escenario de aplicación}

La presente sección se aleja de la anterior abstracción conceptual y propone un escenario sobre el que verificar la idoneidad de modelos complejos de metadatos para abordar realidades documentales igualmente complejas.

El Archivo Municipal de Cartagena gestiona los documentos producidos por el Ayuntamiento del que depende, y que comienzan a datarse en 1246, aunque por supuesto continúan creándose. Estos documentos se generan en su mayor parte en soporte analógico, aunque con el concurso de documentos digitales, sobre todo del tipo bases de datos, que también debieran gestionarse archivísticamente. El Archivo tiene algún grado de control sobre algunos de estos procedimientos de generación de documentos, pero no sobre todos, ni de manera exhaustiva. Además, este control, cuando existe, no está explícitamente declarado, sino que procede más bien de acuerdos más o menos formales y más o menos puntuales con la unidad de informática del Ayuntamiento, el llamado Centro de Proceso de Datos. Como sucede en cualquier otra Administración local, en la generación de documentos del Ayuntamiento de Cartagena intervienen múltiples agentes, tanto internos como externos a la organización, y algunos de ellos con poder decisorio. Dependiendo de las unidades que generan documentos, los procesos que abarcan desde la creación a la conservación definitiva pueden estar reglados o no.

Además, bien que de manera en alto grado inconsciente, el Ayuntamiento genera un alto número de documentos digitales que no están recibiendo ningún tipo de tratamiento archivístico: las bases de datos anteriormente mencionadas, que concurren a la creación de documentos analógicos, sitios web interactivos, miles de correos electrónicos, mensajes SMS, aplicaciones de flujos de tareas, aplicaciones de gestión de recursos humanos, aplicaciones financieras, etc.

Por otra parte, el Archivo, por sí mismo o en cooperación con otras unidades, ha iniciado un conjunto de proyectos de mayor o menor alcance, que también generan documentos analógicos, digitales o ambos. Así, por ejemplo, un proyecto de historia oral que almacena distintos tipos de ficheros digitales, asociados a metadatos en base de datos. El Archivo aloja, por otra parte, distintos fondos personales y colecciones bibliográficas, hemerográficas y visuales; e impulsa la creación de sitios web, algunos de ellos generados mediante herramientas de gestión de contenidos y tecnología wiki. El Archivo mantiene estrechos vínculos, particularmente en lo que se refiere a la innovación tecnológica, con otras unidades informativas y culturales del Ayuntamiento.

Este intratable magma de documentación analógica y digital se describe oficialmente de acuerdo con ISAD $(G)$, aunque esto por supuesto no es cierto: $\operatorname{ISAD}(\mathrm{G})$ resulta tan insuficiente para tantos procesos de asignación de metadatos, que en el curso del tiempo se han ido introduciendo sustanciales adiciones y modificaciones que satisfacen de mejor manera los intereses descriptivos del Archivo. Sin embargo, puesto que ni la insuficiencia de la norma ni su 
muy substancial "re-definición" han sido explícitamente planteadas ni planificadas por el Archivo, tales alteraciones han tenido lugar de manera poco normalizada, y en algunos casos bastante aleatoria, lo que ha conducido a un alto grado de des-integración de las prácticas descriptivas, no sólo entre unidades, sino entre subconjuntos de documentos dentro de una sola unidad. El Archivo se desenvuelve de facto dentro de una perspectiva postcustodial y haciendo uso aleatorio del pensamiento y las herramientas procedentes del continuo de los documentos; sin embargo, la resistencia pretérita a asumir sin timidez estas dependencias conceptuales, ha conducido a esta situación de des-integración que se pretende superar, en primer lugar mediante la mejora y actualización de las tecnologías. Sin embargo, las respuestas meramente tecnológicas, si bien aligeran la situación, no resuelven el problema de la gestión integrada de la información y los documentos, si no se adoptan también medidas relativas a la redefinición de los procedimientos archivísticos. La exploración de la aplicabilidad de modelos complejos de metadatos, tal como RKMS, en sistemas integrados de gestión de información/documentos, de tal manera que se facilite la incorporación interoperable de distintas series de metadatos, está en la base de la segunda fase del proyecto.

\section{Conclusiones}

Hasta el momento, sobre el escenario descrito se ha realizado sobre todo teoría. Una segunda fase del proyecto debiera mostrar que el Archivo, y, más allá, el propio Ayuntamiento, es capaz de manejar un solo sistema de gestión de la información para todos sus conjuntos de documentos, del cual el sistema de gestión de documentos de archivo sería un subsistema con requisitos específicos; este sistema de gestión de información, a su vez, estaría integrado en un sistema más amplio de gestión de conocimiento. Tal conjunto concéntrico de sistemas integrados permitiría llevar a cabo de manera coherente los procesos de gestión de información y documentos, incluído, en lo que nos concierne, el proceso de descripción o asignación de metadatos. Dada la complejidad, tanto del sistema como de los objetos bajo su tratamiento, la presente exposición postula la necesidad de un modelo complejo de metadatos que permita reflejar todas las circunstancias de creación, gestión y uso de los documentos, en diversos ámbitos, dentro y fuera de la organización, y desde diferentes orígenes: documentos administrativos, memoria oral, documentos digitales complejos u objetos informativos no evi- denciales. Si esta aproximación es viable es asunto de otra exposición, en otro lugar del continuo del espacio y el tiempo.

\section{Referencias}

Acland, Glenda; Reed, Barbara; McKemmish, Sue (1999): Documenting Business: The Australian Recordkeeping Metadata Schema. // ADCS'99, Australasian Document Computing Symposium, December 1999. URL: http://www.sims.monash.edu.au/research/rcrg/publicatio ns/adcs.html

Acland, Glenda; Cumming, Kate; McKemmish, Sue (1999): The End of the Beginning: The SPIRT Recordkeeping Metadata Project. // Archives at Risk: Accountability, Vulnerability and Credibility, 1999 Annual Conference of the Australian Society of Archivists, July 1999. URL: http://www.sims.monash.edu.au/research/rcrg/publicatio ns/asaq99.html

Australian Society of Archivists Committee on Descriptive Standards: Describing Archives in Context: A Guide to Australian Practice: Consultation draft, August 2003 (2003). URL: http://www.archivists.org.au/cds/Series\% 20 system $\% 20$ codification $\% 20 \% 20 \% 20$ August $\% 202003$. pdf

Clever Recordkeeping Metadata: Create Once, Use Many Times - The Clever Use of Metadata in eGovernment and eBusiness Processes In Networked Environments (2005). URL: http://www.sims.monash.edu.au/research/ rcrg/research/crm/index.html

Cook, Terry (1994): Documentos electrónicos, mentes de papel: la revolución en gestión de la información y archivos en la era post-custodial y post-moderna. URL: http://archivo.cartagena.es/recursos/texto0_cook_electro nic.pdf

Comeau, Sheila (1998): Encoded Archival Description (EAD) and the Creation of Electronic Finding Aids. // National Library of Canada (December 7, 1998). URL: http://www.nlc-bnc.ca/9/1/p1-257-e.html

Delgado Gómez, Alejandro (2006): El principio de contextualidad y relación de los documentos: una aproximación tentativa. // Ibersid 2006: XI Encuentros Internacionales sobre Sistemas de Información y Documentación. Zaragoza, España, del 1 al 3 de octubre de 2006.

Describing Archives: A Content Standard (2004). Chicago: The Society of American Archivists, 2004

The Society of American Archivists (2003): Statement of Principles for the Custard Project. URL: http://www. archivists.org/news/custardproject.asp

Encoded Archival Context Beta (2004). URL: http://www.iath.virginia.edu/eac/

Encoded Archival Description Version 2002 Official Site (2002). URL: http://www.loc.gov/ead/

Evans, Joanne; McKemmish, Sue; Bhoday, Karuna (2005): Create Once, Use Many Times: The Clever Use of Recordkeeping Metadata for Multiple Archival Purposes. // Archival Science. 5 (2005).

Hensen, Steven (comp.) (1989): Archives, Personal Papers and Manuscripts: a Cataloging Manual for Archival Repositories, Historical Societies and Manuscripts Libraries.. 2nd ed. Chicago: Society of American Archivists, 1989

Hofman, Hans (2005): El archivo. // McKemmish, Sue; Piggott, Michael; Reed, Barbara; Upward, Frank (eds.): Archives: Recordkeeping in Society. Wagga Wagga: Charles Sturt University, Centre for Information Studies, 2005.

Hurley, Chris (2004): Parallel Provenance (If these are your records, where are your stories?). URL: http://www. 
sims.monash.edu.au/research/rcrg/publications/parallelprovenance-combined.pdf

Hurley, Chris (1995): Problems with Provenance. En: Archives and Manuscripts. 23:2 (1995). URL: http:// www.sims.monash.edu.au/research/rcrg/publications/pro venance.html

International Council On Archives, Ad Hoc Commission on Descriptive Standards (1993): ISAD(G) General International Standard Archival Description: Adopted by the Ad Hoc Commission on Descriptive Standards: Stockholm, Sweden, 21-23 January 1993 : (Final ICA approved version) (1993). Ottawa: ICA, 1993.

International Council on Archives, Committee on Best Practices and Professional Standards (2006): ISAF: International Standard For Activities/Functions of Corporate bodies Draft: Developed by the Committee on Best Practices and Professional Standards: Paris, France, May 2006

International Council on Archives, Committee on Descriptive Standards (2004): ISAAR (CPF) (2004): International Standard Archival Authority Record for Corporate Bodies, Persons and Families. 2nd ed.. Paris: ICA, 2004.

International Council On Archives, Committee on Descriptive Standards (2000): ISAD(G): General International Standard Archival Description: Adopted by the Committee on Descriptive Standards Stockholm, Sweden, 19-22 September 1999 (2000). 2nd ed. Ottawa: ICA, 2000.

ISO 23081-1:2006 (2006): Information and documentation -Records management processes -- Metadata for records -- Part 1: Principles (2006). Geneve: International Organization for Standardization, 2006.

ISO 15489-1:2001: Information and documentation -- Records management -- Part 1: General (2001). Geneva: International Organization for Standardization, 2001.

ISO/TR 15489-2:2001: Information and documentation -Records management -- Part 2: Guidelines (2001). Geneva: International Organization for Standardization, 2001.

McKemmish, Sue (1996): Evidencia de mí... URL: http:// archivo.cartagena.es/recursos/texto0_evidencia.pdf

McKemmish, Sue; Acland, Glenda (1999): Accesing Essential Evidence on the Web: Towards an Australian Recordkeeping Metadata Standard. // The Web after a De- cade: Proceedings of AusWeb99, the Fifth Australian World Wide Web Conference, April 1999. URL: http://ausweb.scu.edu.au/aw99/papers/mckemmish/

McKemmish, Sue; Acland, Glenda; Cumming, Kate; Reed, Barbara; Ward, Nigel (1999): Australian Recordkeeping Metadata Schema Version 1.0. URL: http://www. sims.monash.edu.au/research/rcrg/research/spirt/deliver /RKMSGen\%2BTechlntro.pdf

McKemmish, Sue; Acland, Glenda; Reed, Barbara (1999): Towards a Framework for Standardising Recordkeeping Metadata: The Australian Recordkeeping Metadata Schema. // Records Management Journal, UK, 9:3 (1999). URL: http://www.sims.monash.edu.au/research/ rcrg/publications/framewrk.html

McKemmish, Sue; Acland, Glenda; Ward, Nigel; Reed, Barbara (1999): Describing Records in Context in the Continuum: the Australian Recordkeeping Metadata Schema. I/ Archivaria 48 (1999). URL: http://www.sims.monash.edu.au/research/rcrg/publicatio ns/archiv01.htm

McKemmish, Sue; Parer, Dagmar (1998): Towards Frameworks for Standardising Recordkeeping Metadata. // Archives and Manuscripts, 26:1 (1998). URL: http://www.sims.monash.edu.au/research/rcrg/publicatio ns/recordkeepingmetadata/smckrmp1.html

Pitti, Daniel V. (1999): Encoded Archival Description: An Introduction and Overview. // D-Lib Magazine. 5:11 (1999)

Pitti, Daniel V. (1997): Encoded Archival Description: The Development of an Encoding Standard for Archival Finding Aids. // The American Archivist. 60:3 (1997).

Recordkeeping Metadata Project (1999). URL: http:// www.sims.monash.edu.au/research/rcrg/research/spirt/i ndex.html

Reed, Barbara (1997): Metadata: Core record or core business?. // Archives and Manuscripts, 25:2 (1997). URL: http://www.sims.monash.edu.au/research/rcrg/publicatio ns/recordscontinuum/brep1.html

Schauder, Don; Stillman; Larry; Johanson, Graeme (2005): Sustaining a Community Network: The Information Continuum, E-Democracy and the Case of Vicnet. // The Journal of Community Informatics. 1:2 (2005). 